\title{
Simple Method for Screening the Affected Birds with Remarkably Hardened Pectoralis Major Muscles among Broiler Chickens
}

\author{
Takeshi Kawasaki $^{1,2}$, Takashi Yoshida ${ }^{2}$ and Takafumi Watanabe ${ }^{3}$ \\ ${ }^{1}$ Research Office Concerning the Health of Humans and Birds, Masu-ura, Abashiri, Hokkaido 099-3119, Japan \\ ${ }^{2}$ Medical Engineering Course, Graduate School of Engineering, Kitami Institute of Technology, \\ Koen-cho, Kitami, Hokkaido 090-8507, Japan \\ ${ }^{3}$ Faculty of Agriculture, Shinshu University, Minami-minowa, Kami-ina, Nagano 399-4598, Japan
}

\begin{abstract}
Remarkably hardened pectoralis major muscles are increasingly found in broiler chickens in some slaughterhouses in Japan. Based on physical examinations, we selected three affected birds and three control birds from a 43-day-old Ross 308 commercial broiler flock, and examined them biochemically, pathologically, and microbiologically. We found that the presence of degenerative lesions in the pectoralis major muscles was associated with an inability to lift the wings to achieve back-to-back wing contact. As the pectoralis major muscle is a major adductor of the humerus, these degenerative lesions may inhibit the extensibility of the muscle, thereby limiting the range of motion of the humerus. Testing the ability to lift the wing is proposed as a new diagnostic method for detecting affected birds.
\end{abstract}

Key words: broiler, creatine kinase, extensibility, pectoralis major, wooden breast

J. Poult. Sci., 53: 291-297, 2016

\section{Introduction}

Several recent reports have described defects in the quality of breast meat from some broiler chickens. Pale-colored exudative meat has been found in both broiler chickens and turkeys (Woelfel et al., 2002; Owens et al., 2009; Bailey et al., 2015; Velleman and Clark 2015). Smith and Northcutt (2009) reported that this pale-colored exudative avian muscle differed from pale, soft, and exudative porcine meat. Kuttappan et al. (2013b) categorized pale-colored exudative pectoralis major muscles on the basis of visual evaluations and histologic comparisons of the degrees of white striping, reflecting myopathic lesions. The incidence of this novel myopathy of the pectoralis major muscles accompanied by white striation and hardening (referred to as "wooden breast") has markedly increased in Finland within the past 3 years (Sihvo et al., 2014). Furthermore, similar occurrences in fast-growing commercial broiler chickens have recently been reported in other countries (Bailey et al., 2015; Mudalal et al., 2015; Trocino et al., 2015; Velleman and Clark, 2015). Affected birds exhibited hard pectoralis major mus-

Received: March 11, 2016, Accepted: April 30, 2016

Released Online Advance Publication: May 25, 2016

Correspondence: K. Watanabe, Laboratory of Animal Functional Anatomy

(LAFA), Faculty of Agriculture, Shinshu University, Minami-minowa

8304, Kami-ina, Nagano 399-4598, Japan.

(E-mail: yosenabe@shinshu-u.ac.jp) cles, and the phenotype of the muscle hardness was associated with various lesions and inflammation of the pectoralis major muscle tissues (Kuttappan et al., 2013b; Sihvo et al., 2014).

Recently, remarkably hardened pectoralis major muscles have been observed in broiler chickens in some slaughterhouses in Japan (Sasaki et al., 2015). Although it was reported that the phenotypic occurrence of wooden breast can be detected by palpation of the pectoralis major muscles of living birds by experienced producer personnel (Velleman and Clark 2015), there is no other information about the clinical symptoms of affected birds. In this study, we examined the relationships among the characteristic clinical signs, pathological lesions, and biochemical markers of wooden breast. In particular, we focused on the clinical indicators of the condition that may assist in identifying affected birds.

\section{Materials and Methods}

\section{Animals and Physical Examination}

Based on physical examinations, we selected three affected birds (Case Nos. 1-3) and three control birds (Case Nos. 4-6) from a 43-day-old Ross 308 commercial broiler flock. The examination determined the index of hardness of the pectoralis major muscles on palpation. The pectoralis major muscles of the affected birds exhibited a rubbery elasticity, whereas those of the control birds were flexible. 
All birds were observed during standing and walking, and the mobility and extensibility of each wing were tested. After palpation, the posture of each bird was photographed, and the bird was weighed.

\section{Biochemical Examination}

Blood samples were collected from the wing vein of each bird. Plasma samples were prepared by centrifugation of whole blood (using heparin lithium as an anticoagulant; $6,500 \mathrm{rpm} / 2200 \times g$ for $5 \mathrm{~min}$ ) and stored in a $-20^{\circ} \mathrm{C}$ freezer until analysis. The plasma samples were pretreated with 1,3dichloro-1,1,2,2,3-pentafluoropropane (FRIGEN ${ }^{\circledR}$ II; Siemens Healthcare Diagnostics, Malvern, PA, USA), and then diluted with sample diluent (Idexx Laboratories, Westbrook, ME, USA) as necessary. A VetScan VS2 Analyzer (Abaxis, Union City, CA, USA) was used to examine the plasma levels of L-aspartate aminotransferase, bile acid, creatine kinase (CK), uric acid, glucose, calcium, phosphorus, total protein, albumin, globulin, potassium $\left(\mathrm{K}^{+}\right)$, and sodium.

\section{Pathologic Examination}

All birds underwent a postmortem examination immediately after euthanasia. Before laparotomy, swabs were aseptically inserted into a small incision in the skin of the abdomen and wiped across the surface of the pectoralis major muscles with the other swabs for microbiology. The pectoralis major muscles and the legs were removed from each carcass, and weighed individually. The legs were weighed after removal of the skin and feet. The ratios of the weight of the pectoralis major muscles and legs (without skin and feet) to the body weight were calculated as percentages. During necropsy, tissue samples were collected from the pectoralis major muscles, spleen, liver, kidney, and reproductive organs. The pectoralis major muscles were fixed in $10 \%$ formalin, and embedded in paraffin according to standard procedures. Transverse and longitudinal paraffin sections were cut at a thickness of $3 \mu \mathrm{m}$ and mounted on silanecoated glass slides. Deparaffinized transverse and longitudinal sections were subjected to hematoxylin and eosin staining and azan staining. All sections were examined by light microscopy. The shortest diameters of 300 muscle fibers were measured randomly on transverse sections from each pectoralis major muscle.

\section{Microbiological Examination}

Swabs collected from the abdominal incision or from the surface of the pectoralis major muscles were smeared directly on brain-heart infusion agar [brain-heart infusion broth (BD, Franklin Lakes, NJ, USA) combined with agar (Kishida, Osaka, Japan)], XM-G agar (Nissui, Tokyo, Japan), and Staphylococcus Medium No. 110 (Nissui) plates, which were subsequently incubated at $37^{\circ} \mathrm{C}$ for $24-48 \mathrm{~h}$.

\section{Results}

The three affected birds (Case Nos. 1-3) exhibited an abnormal static attitude with regard to their standing position (bent-forward posture, with wings outstretched to achieve balance) (Fig. 1b), but walked actively like the control birds (Fig. 1a). In addition, the affected birds were unable to lift their wings sufficiently to achieve back-to-back wing con- tact, unlike the control birds (Fig. 1c, d). The body weight of the affected birds ranged from 2,920 to 3,560 g, while that of the control birds (Case Nos. 4-6) ranged from 2,760 to 2,970 g. During necropsy, the pectoralis major muscles of the affected birds appeared flatter with a wider surface than those of the control birds (Fig. 2). Varying degrees of white striations parallel to the muscle fibers were evident on the surface of the pectoralis major muscles in the affected birds (Fig. 2b). A mucoid exudate was also visible on the surface of the pectoralis major muscles in Case No. 1 (Fig. 3). No specific lesions were evident macroscopically in the internal organs of any of the cases. The pectoralis major muscle-to-body weight ratio ranged from $8.6 \%$ to $11.1 \%$ in the affected birds and from $8.4 \%$ to $9 \%$ in the control birds. The leg-to-body weight ratio ranged from $8.7 \%$ to $9.9 \%$ in the affected birds and from $7.3 \%$ to $9.4 \%$ in the control birds.

Data for the biochemical indices are presented in Table 1. Notably, CK was elevated in all birds examined in this study. The CK levels in the affected birds ranged from 53,760 to 59,580 U/L, whereas those in Case Nos. 4 and 5 (control birds) were 19,760 and $19,300 \mathrm{U} / \mathrm{L}$, respectively. The CK levels were highest in Case No. 6 (control bird) at 63,800 $\mathrm{U} / \mathrm{L}$. The L-aspartate aminotransferase and $\mathrm{K}^{+}$levels were slightly lower in Case Nos. 4 and 5 than in the other cases.

Microscopically, a wide variation in fiber size was observed in the pectoralis major muscles, reflecting hypertrophy and degeneration of the muscle fibers, in the affected birds. The intermuscular connective tissue had thickened significantly in the affected birds, but appeared normal in the control birds (Fig. 4). The medians of muscle fiber diameter in the control birds (Case Nos. 1-3) were 43.4, 47.1 and 58.0 $\mu \mathrm{m}$, respectively. And those of in the affected birds (Case Nos. 4-6) were $45.0,51.8$ and $55.2 \mu \mathrm{m}$, respectively. The frequency distribution of the diameter of muscle fibers peaked for diameters of $40-70 \mu \mathrm{m}$ in the control birds. In contrast, there was no clearly discernible pattern for the distribution of muscle fiber diameters in the affected birds (Fig. 5). Segmental necrosis of the muscle fibers was evident in all cases, but was more severe in the affected birds. In the affected birds, abnormal long multinuclear chains, fiber splitting, and vacuolar degeneration were observed to varying degrees in longitudinal sections (Fig. 6).

No bacterial growth was observed on any of the media plates in all cases.

\section{Discussion}

In poultry, several cases of degenerative myopathy arising from nutritional deficiencies or infectious diseases have been reported, and these cases have offered insights into the causes of degenerative myopathy (Sihvo et al., 2014). However, a new type of myopathy, referred to as "white striping" or "wooden breast", which occurs without nutritional deficiencies or infectious diseases and only in the pectoralis major muscles of commercial broiler chickens has frequently been reported in recent years in the worldwide broiler industry (Sihvo et al., 2014; Velleman and Clark 2015). The white striping is characterized by visible white lines parallel to the 

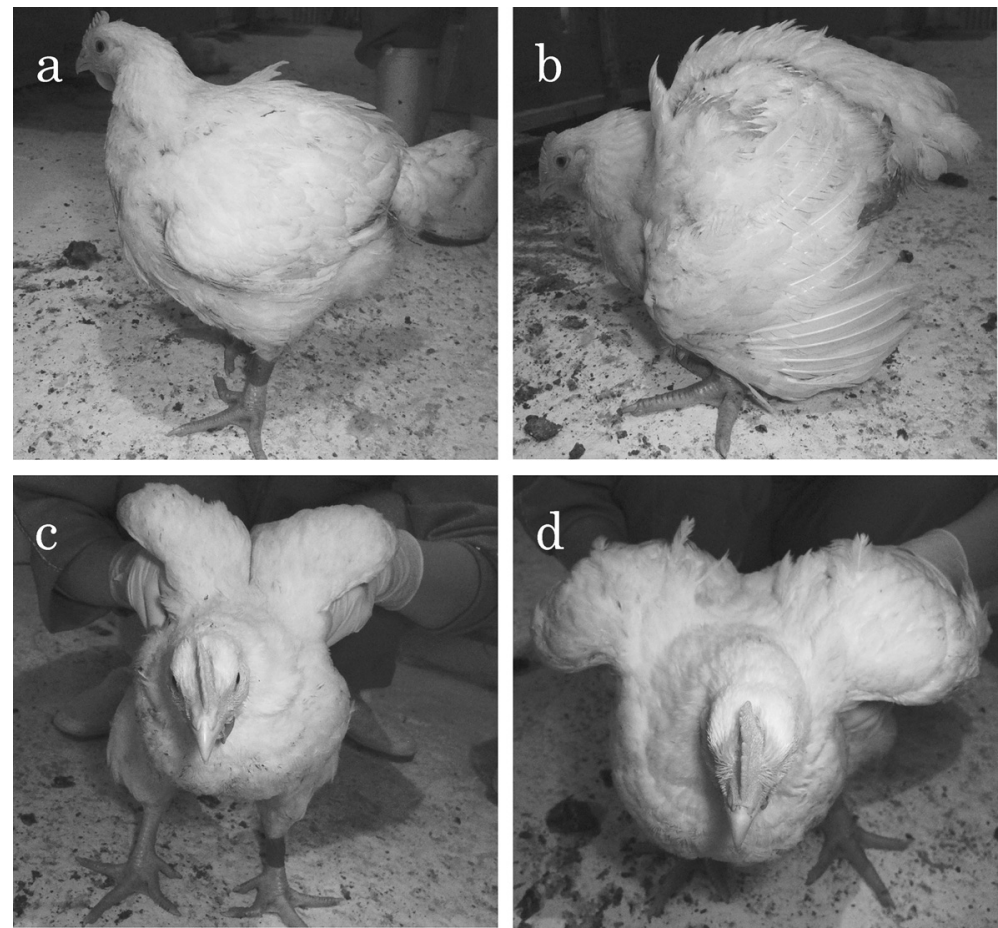

Fig. 1. Standing positions of a control bird (a, c) and an affected bird (b, d). a: Standing position of a control bird (Case No. 4), left-side view. In the resting state, the head is held higher than the vertebral column. b: Standing position of an affected bird (Case No. 2), left-side view. In the resting state, the head is positioned lower than the vertebral column and the wings are outstretched to adjust the center of gravity. c: Standing position of a control bird (Case No. 4), front view. The control bird is able to lift its wings easily to achieve back-to-back wing contact. $\mathrm{d}$ : Standing position of an affected bird (Case No. 2), front view. The affected bird is unable to lift its wings sufficiently to achieve back-toback wing contact.
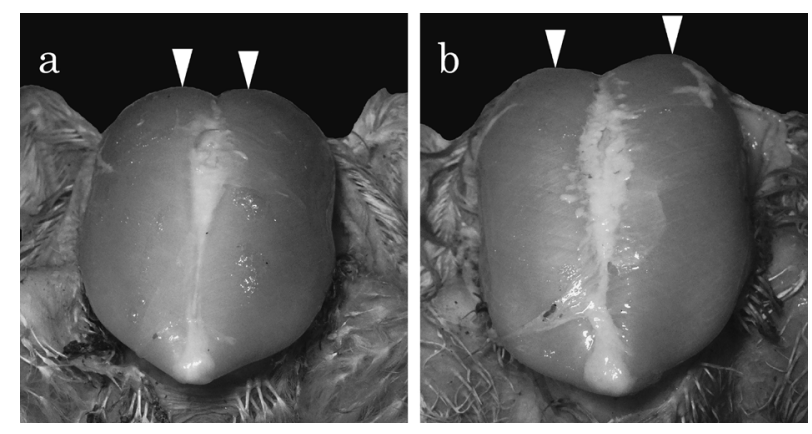

Fig. 2. Distance between the vertices of the pectoralis major muscles in the ventral aspect of a control bird (Case No. 4) and an affected bird (Case No. 2). The distance in the affected bird (b) is greater than that in the control bird (a).

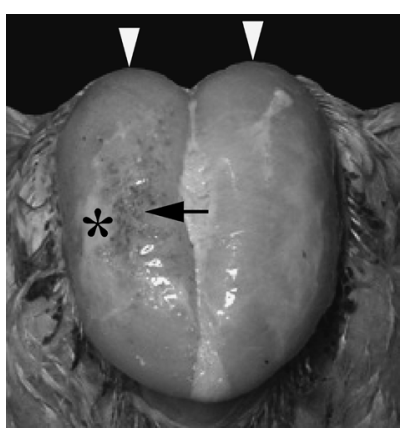

Fig. 3. Surface view of the pectoralis major muscles in an affected bird (Case No. 1). A mucoid exudate (asterisk), petechiae (arrow), and bulging (arrow heads) are visible on the surface of the pectoralis major muscles. 
Table 1. Weights of the body, pectoralis major muscles, and legs and biochemical indices of plasma samples in the control birds (Case Nos. 4-6) and affected birds (Case Nos. 1-3)

\begin{tabular}{|c|c|c|c|c|c|c|}
\hline \multirow[b]{2}{*}{ Case No. } & \multicolumn{3}{|c|}{ Control } & \multicolumn{3}{|c|}{ Affected } \\
\hline & 4 & 5 & 6 & 1 & 2 & 3 \\
\hline Sex & male & female & female & male & male & female \\
\hline Body weight (g) & 2,970 & 2,760 & 2,770 & 3,025 & 3,560 & 2,920 \\
\hline PmM weight (g) & 249 & 232 & 248 & 335 & 306 & 271 \\
\hline Leg weight (g) & 280 & 250 & 203 & 300 & 345 & 255 \\
\hline $\mathrm{AST}(\mathrm{U} / \mathrm{L})$ & 346 & 300 & 553 & 635 & 588 & 595 \\
\hline BA (Umol/L) & $<35$ & $<35$ & $<35$ & $<35$ & $<35$ & $<35$ \\
\hline $\mathrm{CK}(\mathrm{U} / \mathrm{L})$ & 19,760 & 19,300 & 63,800 & 54,720 & 53,760 & 59,580 \\
\hline UA (mg/dL) & 2.3 & 3.1 & 6.2 & 3.5 & 3.7 & 5.2 \\
\hline GLU (mg/dL) & 230 & 251 & 236 & 236 & 215 & 243 \\
\hline $\mathrm{Ca}(\mathrm{mg} / \mathrm{dL})$ & 12 & 11.5 & 12.2 & 12.2 & 11.6 & 12.6 \\
\hline PHOS (mg/dL) & 6.4 & 6.3 & 6.8 & 6.7 & 7.6 & 6.6 \\
\hline $\mathrm{TP}(\mathrm{g} / \mathrm{dL})$ & 3.8 & 3.8 & 4.1 & 4.2 & 3.5 & 4.6 \\
\hline $\operatorname{ALB}(\mathrm{g} / \mathrm{dL})$ & 2.3 & 2.4 & 2.6 & 2.8 & 2.1 & 2.9 \\
\hline GLOB $(\mathrm{g} / \mathrm{dL})$ & 1.5 & 1.4 & 1.4 & 1.4 & 1.4 & 1.7 \\
\hline $\mathrm{K}^{+}(\mathrm{mmol} / \mathrm{L})$ & 8.1 & 8.6 & 11 & 10.5 & 10.7 & 10.5 \\
\hline $\mathrm{Na}^{+}(\mathrm{mmol} / \mathrm{L})$ & 145 & 144 & 145 & 147 & 145 & 148 \\
\hline
\end{tabular}

Abbreviations: AST, L-aspartate aminotransferase; BA, bile acid; CK, creatine kinase; UA, uric acid; GLU, glucose; Ca, calcium; PmM, pectoralis major muscle; PHOS, phosphorus; TP, total protein; ALB, albumin; GLOB, globulin; $\mathrm{K}^{+}$, potassium; $\mathrm{Na}^{+}$, sodium.
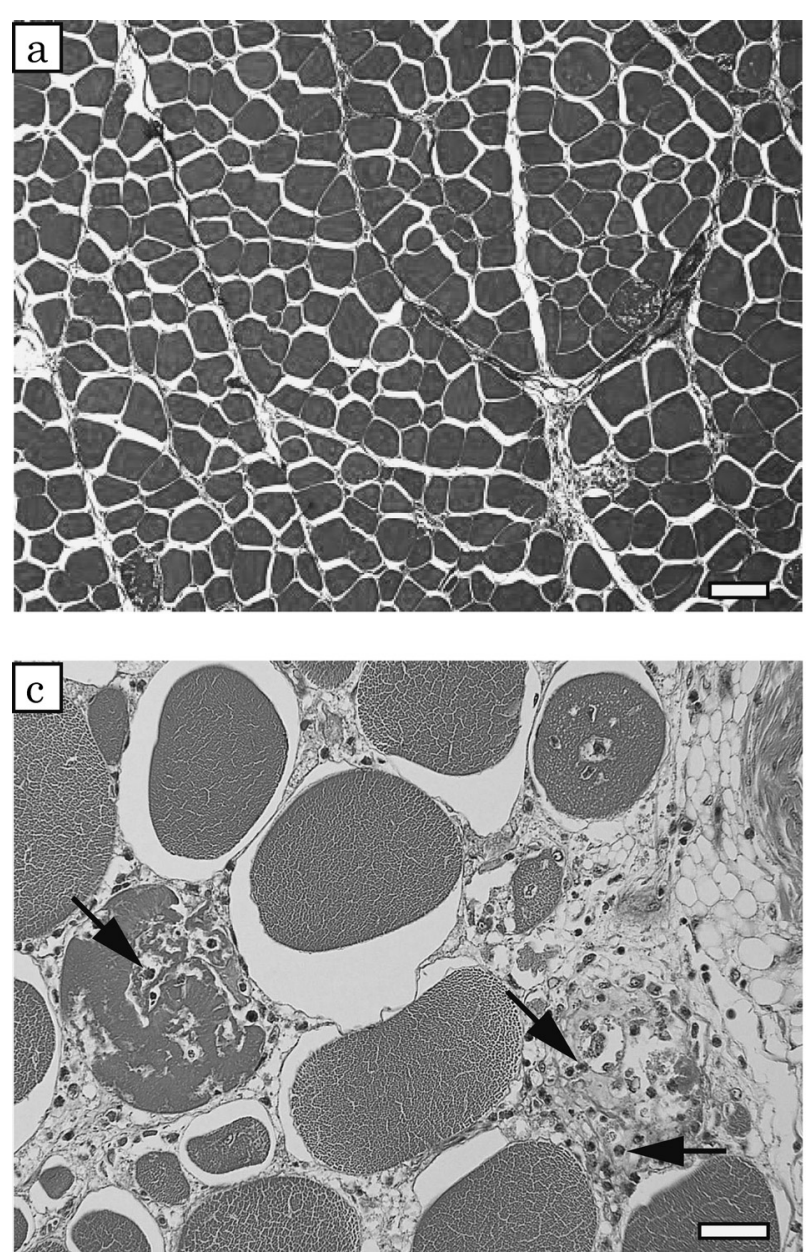

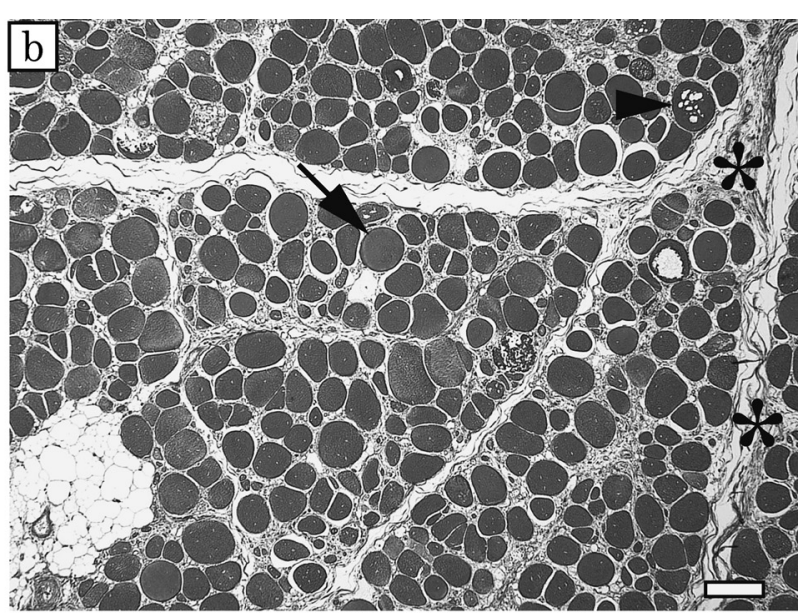

Fig. 4. Cross-sections of the pectoralis major muscles in a control bird (Case No. 5) (a) and an affected bird (Case No. 2) (b), azan staining. a: In the control bird, many muscle fibers show a polygonal shape. $b$ : In the affected bird, there are some round-shaped muscle fibers that show hypertrophy (arrow) and degeneration (arrowhead), and the intermuscular connective tissue shows significant thickening (asterisks). A group of white cell at the lower left corner is adipocytes. Bars $=100 \mu \mathrm{m}$

c: high magnification of cross-sections of pectoralis major muscles in an affected bird (Case No. 2), a hematoxylin eosin staining. A lot of invasive cells (arrows) are observed in degenerated muscle fiber. $B a r=30 \mu \mathrm{m}$ 

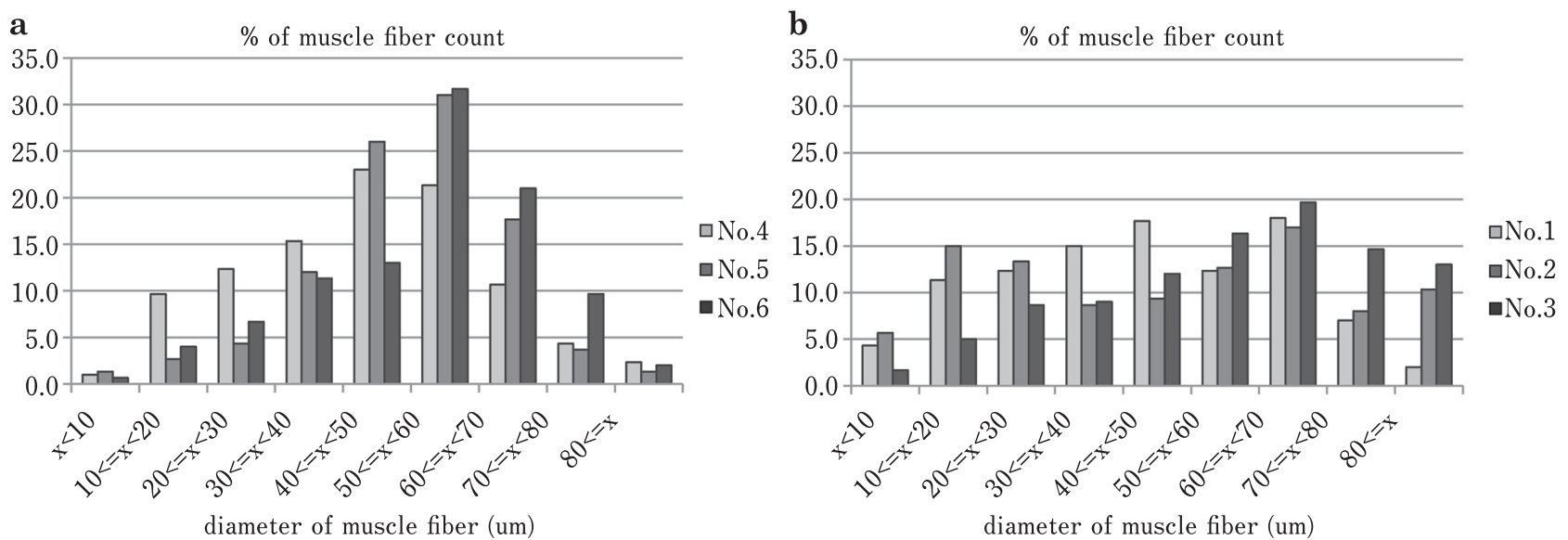

Fig. 5. Diameter distribution ratios of $\mathbf{3 0 0}$ muscle fibers in the control birds (Case Nos. 4-6) (a) and the affected birds (Case Nos. 1-3) (b).
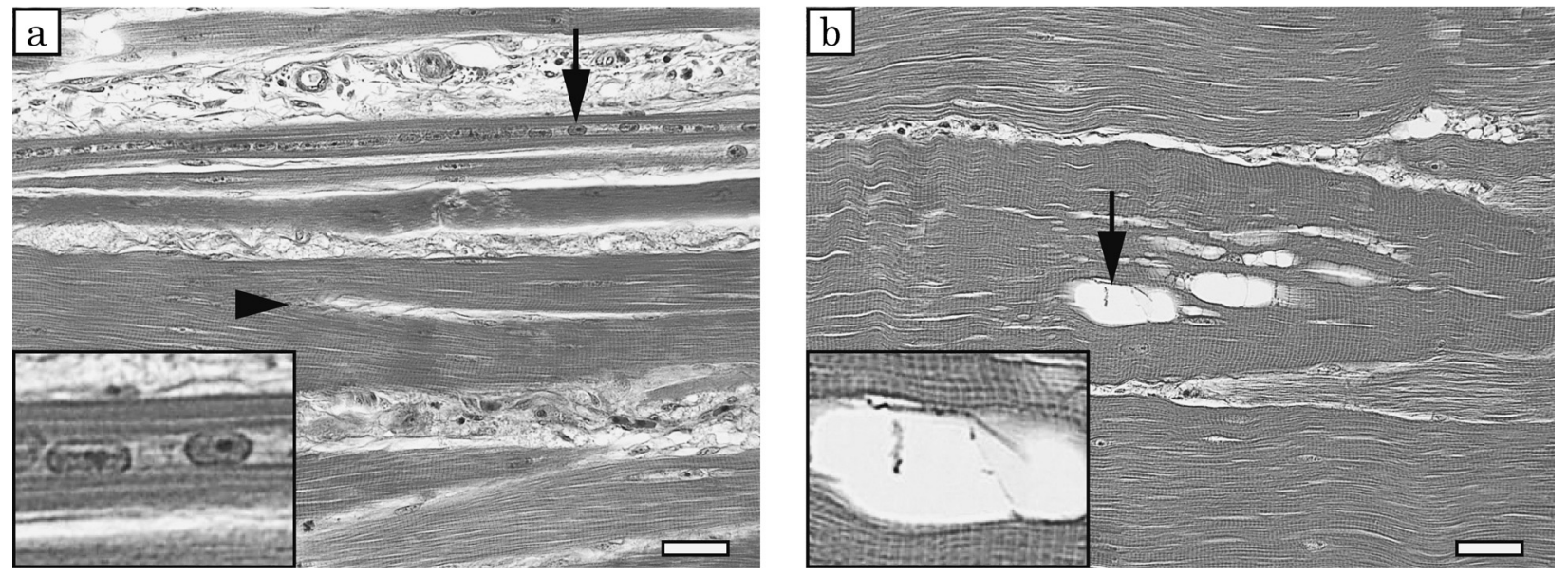

Fig. 6. Longitudinal sections of pectoralis major muscles in affected birds, hematoxylin and eosin staining. a: Long multinuclear chains (arrow) and splitting fibers (arrowhead) are observed in an affected bird (Case No. 1). b: Vacuolar degeneration (arrow) is observed in an affected bird (Case No. 3). Enlarged figures of long multinuclear chains and vacuolar degeneration are inserted in the lower left corner of each figure. $B$ ars $=30 \mu \mathrm{m}$

direction of the muscle fibers, while the wooden breast is characterized by hardening and an exudate of the breast muscle (Bailey et al., 2015). However, the difference in pathogenesis between the two phenotypes has not been strictly classified. Although a mucoid exudate was only observed in Case No. 1, hardened pectoralis major muscles and remarkable fibrosis accompanied by degenerative muscle fibers were observed in the affected birds in the present study. Therefore, we considered that all of the affected birds had wooden breast.

In recent years, white striping or wooden breast has been a hot topic in the Japanese poultry industry. Noticeable wooden breast is discarded or reduced in commercial value by its adverse impact on the purchasing desire of consumers. This is the first paper to report the relationships between wooden breast and clinical symptoms in living birds. Petracci and Cavani (2012) stated that white striping or striations in poultry breast fillets represented an emerging meat quality problem. Kuttappan et al. (2012) revealed that as the degree of white striping increased, there was a significant increase in the occurrence of chronic myopathic lesions along with lipidosis and fibrosis. Sihvo et al. (2014) 
performed more detailed histopathological investigations of wooden breast. They reported that several muscle fibers of wooden breast were degenerated and infiltrated by inflammatory cells, mainly heterophils and macrophages. They also reported that the myodegeneration with regeneration, as well as accumulation of interstitial connective tissue with inflammatory cells, was seen in all affected birds. Moreover, Velleman and Clark (2015) showed overall morphologic assessment of wooden breast including with the gene expression of MYOD1, myogenin, decorin, TGF- $\beta$ and myostatin. Although many pathological studies of wooden breast have been reported, methods for detecting wooden breast in living birds are limited to experienced producer personnel. Furthermore, these reports did not clarify the relationships between clinical symptoms and histopathologic findings.

In the present study, all affected birds, regardless of the degree of wooden breast, exhibited a bent-forward posture with outstretched wings and possessed remarkably hardened pectoralis major muscles. In addition, histopathologic analysis of the pectoralis major muscles tissues of the affected birds revealed mainly hypertrophy and degeneration of muscle fibers, and intermuscular connective tissue thickening. These findings in Japan were consistent with the histologic findings of Sihvo et al. (2014).

Kuttappan et al. (2013a) reported that breast muscle damage evident by severe white striping was associated with increased serum levels of CK, ALT, L-aspartate aminotransferase, and lactate dehydrogenase. Biochemical analyses of plasma samples revealed that the CK levels were high in the affected birds in the present study. In domestic chickens, it was reported that the development of the perimysium of the pectoralis muscle was slower than that of the thigh muscles (Nakamura et al., 2004; Roy et al., 2006). Furthermore, in fast-growing birds, improvements in growth rate and breast meat yield have resulted in further space reduction of the perimysium of the pectoralis muscle (Velleman et al., 2003). The perimysium is a connective tissue that contains blood capillaries (Watanabe et al., 2015). Velleman and Clark (2015) suggested that limiting the available space for capillaries reduced the amount of lactic acid removed from the muscle. They also hypothesized that lactic acid produced from anaerobic respiration was not efficiently removed, resulting in a decreased $\mathrm{pH}$ and muscle damage in the pectoralis major muscles in wooden breast. As a result, this muscle damage was considered to be associated with the increased CK levels. However, the highest CK levels were evident in one of the control birds (Case No. 6). In the present flock, the CK levels in all cases examined were approximately 2-9 times higher than the normal levels in broiler chickens (Tao et al., 2011). And those were also 3-10 times higher than our personal data (CK value range of 43-46 day old broiler normal flocks; 2389-19743 U/L, median $=6637 \mathrm{U} / \mathrm{L}, n=24)$. Therefore, we suspect that the entire flock was particularly susceptible to defective pectoralis major muscle formation. It is possible that Case No. 6 may have been selected just before the onset of wooden breast.
In addition, we revealed that the presence of the lesions was accompanied by an inability to lift the wings sufficiently to achieve back-to-back wing contact. The pectoralis major muscle is a major adductor muscle of the humerus. The degenerative lesions present in the pectoralis major muscle may inhibit their extensibility, in turn limiting the range of motion of the humerus. Testing the ability of chickens to lift their wings may be a useful screening technique for identifying affected chickens regardless of the degree of wooden breast, in a flock. However, this suggestion is not without limitations. Although this study demonstrates an easy objective method to explore wooden breast, without requiring experienced producer personnel, the number of samples in the present study was small. A large number of samples should be used to refine this diagnostic method, and it may be possible to score the wooden breast condition in living animals in the future.

\section{References}

Bailey RA, Watson KA, Bilgili SF and Avendano S. The genetic basis of pectoralis major myopathies in modern broiler chicken lines. Poultry Science, 94: 2870-2879. 2015.

Kuttappan VA, Brewer VB, Apple JK, Waldroup PW and Owens $\mathrm{CM}$. Influence of growth rate on the occurrence of white striping in broiler breast fillets. Poultry Science, 91: 26772685. 2012.

Kuttappan VA, Huff GR, Huff WE, Hargis BM, Apple JK, Coon C and Owens CM. Comparison of hematologic and serologic profiles of broiler birds with normal and severe degrees of white striping in breast fillets. Poultry Science, 92: 339-345. 2013a.

Kuttappan VA, Shivaprasad HL, Shaw DP, Valentine BA, Hargis BM, Clark FD, McKee SR and Owens CM. Pathological changes associated with white striping in broiler breast muscles. Poultry Science, 92: 331-338. 2013b.

Mudalal S, Lorenzi M, Soglia F, Cavani C and Petracci M. Implications of white striping and wooden breast abnormalities on quality traits of raw and marinated chicken meat. Animal, 9: 728-734. 2015.

Nakamura YN, Iwamoto H, Shiba N, Miyachi H, Tabata S and Nishimura S. Growth changes of the collagen content and architecture in the pectoralis and iliotibialis lateralis muscles of cockerels. British Poultry Science, 45: 753-761. 2004.

Owens CM, Alvarado CZ and Sams AR. Research developments in pale, soft, and exudative turkey meat in North America. Poultry Science, 88: 1513-1517. 2009.

Petracci $\mathrm{M}$ and Cavani C. Muscle growth and poultry meat quality issue. Nutrients, 4: 1-12. 2012.

Roy BC, Oshima I, Miyachi H, Shiba N, Nishimura S, Tabata S and Iwamoto H. Effects of nutritional level on muscle development, histochemical properties of myofibre and collagen architecture in the pectoralis muscle of male broilers. British Poultry Science, 47: 433-442. 2006.

Sasaki Y, Sato R and Oka Y. Causes and Incidence of superficial pectoral myopathy in broiler chickens at a processing plant in Iwate prefecture. Journal of the Japanese Society on Poultry Disease (In Japanese), 51: 99-105. 2015.

Sihvo HK, Immonen $\mathrm{K}$ and Puolanne E. Myodegeneration with fibrosis and regeneration in the pectoralis major muscle of broilers. Veterinary Pathology, 51: 619-623. 2014. 
Smith DP and Northcutt JK. Pale poultry muscle syndrome. Poultry Science, 88: 1493-1496. 2009.

Tao X, Dong H, Zhang H and Xin H. Sex-based responses of plasma creatine kinase in broilers to thermoneutral constant and cyclic high temperatures. British Poultry Science, 52: 800-806. 2011.

Trocino A, Piccirillo A, Birolo M, Redaelli G, Bertotto D, Filiou E, Petracci M and Xiccato G. Effect of genotype, gender and feed restriction on growth, meat quality and the occurrence of white striping and wooden breast in broiler chickens. Poultry Science, 94: 2996-3004. 2015.

Velleman SG, Anderson JW, Coy CS and Nestor KE. Effect selection for growth rate on muscle damage during turkey breast muscle development. Poultry Science, 82: 1069-1074. 2003.
Velleman SG and Clark DL. Histopathologic and myogenic gene expression changes associated with wooden breast in broiler breast muscles. Avian Diseases, 59: 410-418. 2015.

Watanabe T, Nishimura K, Takeuchi R, Koyama Y, Kusubata M, Takehana $\mathrm{K}$ and Hiramatsu K. Oral ingestion of collagen peptide causes change in width of the perimysium of the chicken iliotibialis lateralis muscle. Journal of Veterinary Medical Science, 77: 1413-1417. 2015.

Woelfel RL, Owens CM, Hirschler EM, Martines-Dawson R and Sams AR. The characterization and incidence of pale, soft, and exudative broiler meat in a commercial processing plant. Poultry Science, 81: 579-584. 2002. 